\title{
Um circuito simples com bomba única para circulação extracorpórea com oxigenação autógena
}

\author{
Euclydes MARQUES*, Ismar N. CESTARI, Idágene A. CESTARI e Adolfo A. LEIRNER*
}

RBCCV 44205-586

Marques E, Cestari I N, Cestari I A, Leirner A A - Um circuito simples com bomba única para circulação extracorpórea com oxigenação autógena. Rev Bras Cir Cardiovasc 2002; 17(2): 52-57.

RESUMO: Material e Métodos: Foi testado em 30 cães um circuito capaz de promover circulação extracorpórea (CEC) com oxigenação autógena (OA) do sangue, usando apenas uma bomba centrífuga. Esta montagem dispensou bombeamento para o lado direito: o gradiente de pressão bastante para vencer a resistência arterial pulmonar foi vencido aumentando-se a pressão nas artérias pulmonares pela expansão da volemia e diminuindo-se a pressão do átrio esquerdo pela drenagem dessa câmara mediante um sifão. O coração foi mantido em ritmo de fibrilação ventricular durante o período de perfusão e ao seu término, o ritmo próprio foi recuperado mediante cardioversão elétrica.

Resultados: Este circuito permitiu a manutenção de parâmetros hemodinâmicos e gases sangüíneos adequados durante a perfusão. O campo operatório e a mobilidade do coração foram similares aos proporcionados pela CEC convencional.

Conclusão: Concluímos que o uso de bomba centrífuga única simplifica a $O A$, podendo tornar-se uma escolha prática nos procedimentos de revascularização do miocárdio.

DESCRITORES: Circulação Extracorpórea, métodos. Procedimentos cirúrgicos cardíacos, métodos.

\section{INTRODUÇÃO}

Durante cerca de quarenta anos, a circulação extracorpórea (CEC) tem sido habitualmente utilizada em cirurgia cardíaca. Nos anos cinqüenta foram feitas muitas tentativas no intuito de oxigenar o sangue à custa dos próprios pulmões do operando ${ }^{(1-6)}$. Todavia, nos casos em que a cardiotomia era obrigatória, as dificuldades técnicas tornaram complicado e arriscado o uso da oxigenação autógena (OA). A CEC com exclusão do coração e dos pulmões foi adotada rotineiramente para as cirurgias cardíacas a céu aberto.

Durante os anos 70, a revascularização do miocárdio tornou-se a operação mais freqüentemente executada sobre o coração e o mesmo método de CEC continuou sendo adotado. Entretanto, sendo esta intervenção um procedimento realizado com o coração fechado, o emprego da OA foi novamente levado em conta por vários pesquisadores que demonstraram e divulgaram suas vantagens sobre a CEC convencional (7-12). No entanto, essa técnica não se tornou uma rotina universal, provavelmente porque algumas dificuldades inerentes ao método sobrepujavam suas vantagens.

Analisando a literatura, concluímos que uma considerável inconveniência do método é a dificuldade de compatibilizar os débitos das duas bombas empregadas para garantir a grande e a pequena circulação. O objetivo dos experimentos aqui relatados foi evitar esta inconveniência, utilizando-se apenas uma bomba sustentando a circulação sistêmica. $O$ gradiente de pressão transpulmonar responsável pela

Trabalho realizado no Instituto do Coração do Hospital das Clínicas da Faculdade de Medicina da Universidade de São Paulo. São Paulo, SP, Brasil.

*Do Instituto do Coração.

Endereço para correspondência: Euclydes Marques. Instituto do Coração. Divisão de Bioengenharia. Av. Dr. Enéas de Carvalho Aguiar, 44. CEP: 05403-000 São Paulo, SP, Brasil. Tel: (11) 3069-5262. 
perfusão através dos pulmões foi vencido aumentando-se a pressão no átrio direito por expansão volêmica e diminuindo-se a pressão do átrio esquerdo pela sua drenagem, coletando-se o sangue das veias pulmonares em um reservatório situado abaixo do nível do coração, o que gera um efeito de sifão.

\section{MATERIAL E MÉTODOS}

Foram executadas 30 perfusões experimentais usando-se cães mestiços com peso corpóreo entre 13 e $22 \mathrm{~kg}$. Os 20 primeiros experimentos foram realizados com o intuito de padronizar a técnica e estabelecer um protocolo de procedimento (experimentos-piloto). Todos os outros 10 experimentos obedeceram este protocolo e forneceram os dados expostos e analisados adiante.

Os animais foram anestesiados com pentobarbital (15 mg/kg), intubados e colocados em ventilação mecânica (12 ciclos $/ \mathrm{min}$; volume corrente = $25 \mathrm{ml} /$ kg; relação inspiração/expiração de 0.5; $\mathrm{FiO}_{2}$ de 40\%). Os cães foram divididos em dois grupos iguais, ventilados sem ou com pressão expiratória final positiva (PEEP), resultando pressões intratraqueais médias de $6 \mathrm{~cm} \mathrm{H} \mathrm{H}_{2} \mathrm{O}$ e 13,5 $\mathrm{cm} \mathrm{H}_{2} \mathrm{O}$, respectivamente. $\mathrm{O}$ débito cardíaco foi medido pelo método da termodiluição antes e depois da CEC. Foram registrados o eletrocardiograma, as pressões dos gases sangüíneos e as pressões na aorta, na artéria pulmonar e nos átrios. A temperatura do animal foi mantida entre $33 \mathrm{e}$ $35^{\circ} \mathrm{C}$ mediante um trocador de calor instalado no circuito extracorpóreo.

\section{Estabelecimento da CEC}

Foi praticada esternotomia mediana, com abertura do pericárdio, seguida da administração endovenosa de 400 UI de heparina/kg de peso. Uma cânula foi introduzida no átrio esquerdo e ligada a um reservatório com trocador de calor mantido abaixo do nível do animal. O sangue proveniente deste reservatório foi injetado na aorta mediante uma bomba centrífuga (Figura 1). O circuito extracorpóreo foi preenchido com $400 \mathrm{ml}$ de sangue homólogo. Após estabelecer a CEC com oxigenação autógena, o coração foi mantido em ritmo de fibrilação ventricular, sendo transfundido sangue adicional suficiente para manter os parâmetros hemodinâmicos basais e o fluxo na bomba. Este volume adicional necessário foi de aproximadamente $1 \%$ do peso corpóreo. A CEC foi mantida durante 90 minutos, com medidas realizadas a cada 15 minutos. Ao término da experiência, o ritmo próprio do coração

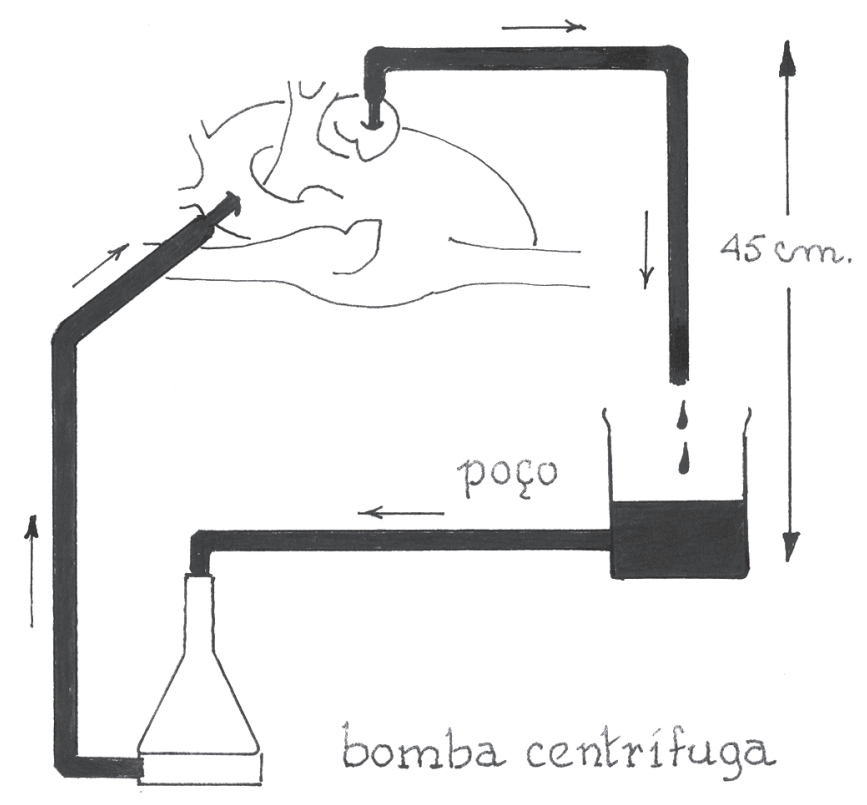

Fig. 1 - Esquema do circuito. O sangue é drenado do átrio esquerdo até um reservatório (poço) situado $45 \mathrm{~cm}$ abaixo de onde é injetado na aorta mediante uma bomba centrífuga. 
foi restabelecido com cardioversão elétrica. Estabelecido o equilíbrio hemodinâmico fora de CEC, foram realizadas medidas finais e os animais foram sacrificados.

\section{RESULTADOS}

O Gráfico 1 mostra os valores de $\mathrm{pO}_{2}$ e $\mathrm{pCO}_{2}$ no sangue arterial, em tempos diferentes, em animais sem PEEP (A) e com PEEP (B). Foi observada uma queda inicial no valor da $\mathrm{pO}_{2}$ em ambos os grupos, de $211.8 \pm 18.6 \mathrm{mmHg}$ e $218.75 \pm 26 \mathrm{mmHg}$ em A e B, durante a condição basal para $147.8 \pm 45.6 \mathrm{mmHg}$ (69.9\% de valor basal) e $190.8 \pm 41.7 \mathrm{mmHg}(87.2 \%$ de valor basal) depois de 15 minutos de OA, respectivamente. Foi observada uma pequena diminuição desses valores durante os 75 minutos restantes do procedimento, permanecendo entretanto os mesmos, dentro de níveis aceitáveis para oxigenação do tecido. No grupo com PEEP o valor médio de $\mathrm{pO}_{2}$ ao término da experiência foi de $152.8 \pm 53.7 \mathrm{mmHg}$.

\section{GRÁFICO 1 \\ COMPORTAMENTO DOS GASES DO SANGUE DURANTE A CIRCULAÇÃO EXTRACORPÓREA (CEC) COM OXIGENAÇÃO AUTÓGENA}
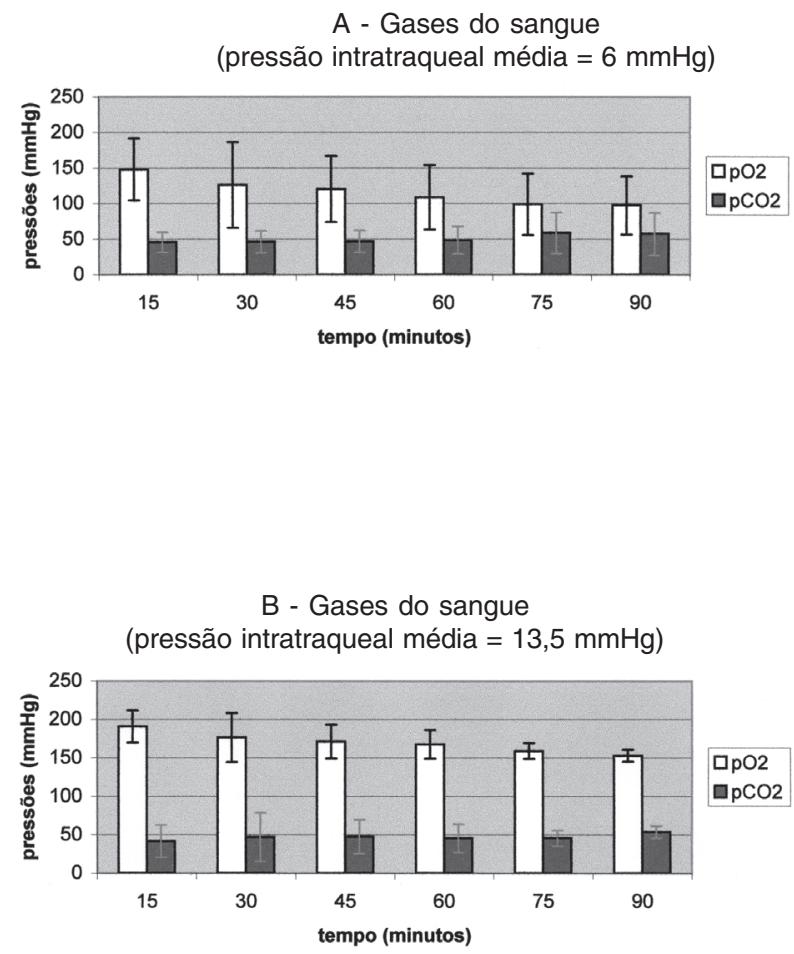

As Tabelas 1 e 2 mostram os valores de índice cardíaco obtidos antes e depois da CEC. A relação do valor final / valor inicial é mostrada na terceira coluna e representa a porcentagem do índice cardíaco encontrada ao término da CEC. Os resultados obtidos com maior pressão traqueal mostram uma menor alteração do índice cardíaco. Entretanto, a análise com teste t não revelou que nenhuma destas alterações fosse estatisticamente significante para $p=0,01$.

TABELA 1. VARIAÇÃO DO ÍNDICE CARDÍACO COM A CIRCULAÇÃO EXTRACORPÓREA (CEC) COM OXIGENAÇÃO AUTÓGENA - ANIMAIS COM PRESSÃO TRAQUEAL MÉDIA DE $6 \mathrm{mmHg}$

\begin{tabular}{lccc}
\hline ANIMAL & ÍNDICE PRÉ-CEC & ÍNDICE PÓS-CEC & VARIAÇÃO \\
\hline 1 & 6,54 & 5,69 & 86,96 \\
\hline 2 & 6,59 & 5,66 & 85,92 \\
\hline 3 & 5,15 & 5,50 & 106,80 \\
4 & 6,39 & 5,95 & 93,18 \\
5 & 8,80 & 7,84 & 89,13 \\
MÉDIAS & 6,69 & 6,13 & 92,40 \\
DESVIOS & 1,32 & 0,97 & 8,52 \\
\hline
\end{tabular}

TABELA 2. VARIAÇÃO DO ÍNDICE CARDÍACO COM A CIRCULAÇÃO EXTRACORPÓREA (CEC) COM OXIGENAÇÃO AUTÓGENA - ANIMAIS COM PRESSÃO TRAQUEAL MÉDIA DE $13,5 \mathrm{mmHg}$

\begin{tabular}{lccc}
\hline ANIMAL & ÍNDICE PRÉ-CEC & ÍNDICE PÓS-CEC & VARIAÇÃO \\
\hline 1 & 6,79 & 6,63 & 97,74 \\
2 & 8,46 & 8,00 & 94,65 \\
3 & 7,38 & 7,38 & 100,00 \\
4 & 9,15 & 8,75 & 95,59 \\
5 & 6,41 & 6,37 & 99,29 \\
MÉDIAS & 7,64 & 7,43 & 97,46 \\
DESVIOS & 1,15 & 0,98 & 2,31 \\
\hline
\end{tabular}



autógena. Rev Bras Cir Cardiovasc 2002; 17(2): 52-57.

O posicionamento do reservatório foi determinado durante experimentos-piloto, variando-se o nível entre o reservatório e o átrio até que se observasse boa drenagem. $O$ reservatório teve que ser colocado a pelo menos $15 \mathrm{~cm}$ abaixo do nível do coração, para que ocorresse drenagem de sangue suficiente. Se o reservatório fosse elevado acima desse nível, a drenagem de sangue ficava prejudicada tornando-se insuficiente para manter o fluxo necessário. Este fluxo cessava completamente se o reservatório fosse elevado até o nível do átrio esquerdo. Um nível de $45 \mathrm{~cm}$ abaixo desta câmara foi considerado adequado e tecnicamente fácil de ser obtido, sendo então adotado para todos os experimentos.

Estando bem drenado o átrio esquerdo, não foi onservada distensão do respectivo ventrículo.

Todos os dez animais retornaram ao ritmo sinusal após a cardioversão elétrica e somente pequenas alterações não específicas do segmento ST foram observadas no eletrocardiograma.

\section{COMENTÁRIOS}

Durante os primórdios da evolução da CEC foram praticadas operações cardíacas utilizando-se os pulmões do paciente para oxigenação sangüínea, com procedimentos com e sem oxigenadores testados simultaneamente. Em 1952, WESOLOWSKI \& WELCH (6) descreveram com pormenores a técnica para OA.

Em 1956, o principal obstáculo para emprego da $\mathrm{OA}$ foi atribuído à dificuldade de coletar o fluxo venoso que sai do pulmão estando o coração aberto ${ }^{(13)}$. Mesmo assim, durante os anos 50, vários grupos executaram circulações extracorpóreas experimentais e clínicas utilizando os pulmões como oxigenadores ${ }^{(1,2,4,14)}$. Em 1960, SHABETAI ${ }^{(5)}$ publicou uma revisão do estado da arte em OA, considerando-a uma interessante abordagem para a cirurgia cardíaca. Entre nós, há mais de 30 anos, o professor Domingos A. Moraes defende a oxigenação autógena em CEC, proclamando "o sangue e o endotélio formam um casamento indissolúvel" (9).

As dificuldades em coletar sangue das veias pulmonares e o perigo de embolia gasosa, em razão da cardiotomia, praticamente impediram o uso rotineiro desta técnica nas operações realizadas para tratar valvopatias e anomalias congênitas, já que em todas elas abriam-se as cavidades cardíacas. Tais inconvenientes sobrepujavam as vantagens proporcionadas pela OA. Por outro lado, o desenvolvimento de oxigenadores descartáveis, disponíveis comercialmente, tornou a oxigenação artificial sobremaneira mais prática. A CEC total firmou-se como rotina para as operações cardíacas a céu aberto.

Nos anos 70, a cirurgia das artérias coronárias amadureceu constituindo a maioria das operações cardíacas sendo a CEC imediatamente utilizada nesse procedimento, pois proporcionava a imobilidade do coração e permita a aplicação de métodos padroniza- dos de proteção do miocárdio. Todavia, logo se levou em conta que estas duas situações podiam ser obtidas sem a exclusão dos pulmões, uma vez que as operações de revascularização não exigem cardiotomia. Poder-se-ia, portanto, auferir as vantagens do emprego da OA sem os seus maiores inconvenientes, ou seja, a dificuldade de drenar o coração aberto e o risco de embolia gasosa.

As vantagens potenciais da exclusão dos oxigenadores artificiais são:

- Os pulmões são mantidos perfundidos, o que Ihes permite atuar nos mecanismos homeostáticos e no balanço da prostaglandina e do tromboxane ${ }^{(15,16)}$.

A perfusão pulmonar evita anóxia tecidual, que favorece a agregação leucoplaquetária e obstrução capilar, predispondo à angústia respiratória ${ }^{(17)}$.

A OA evita o estabelecimento da grande superfície de contato do sangue com material estranho como ocorre nos oxigenadores. Sabe-se que desse contato decorre a ativação de complemento e daí deficiência pulmonar com diminuição da função plaquetária (18-20). O fenômeno se dá tanto com o emprego oxigenadores de membrana como nos de bolhas ${ }^{(16)}$.

A OA pode auxiliar na diminuição de transfusões de sangue, quando comparada a circuitos com oxigenadores, devido à menor redução no número a na função das plaquetas ${ }^{(8)}$.

Em casos de falência cardíaca pós-perfusional, a assistência do ventrículo esquerdo pode ser efetuada facilmente, utilizando-se as cânulas já inseridas e o mesmo circuito da CEC.

A eliminação do oxigenador pode reduzir o custo das operações de revascularização do miocárdio. Considerando o número de operações atualmente realizadas, isto pode representar uma economia significativa, especialmente em países em desenvolvimento.

A maioria dos trabalhos publicados sobre OA descreve métodos onde se utilizam duas bombas e dois reservatórios abertos $(1,2,4,14)$. O emprego de dois reservatórios abertos apresenta o problema de balanceamento de seus níveis com o perigo de introduzir-se ar na circulação se qualquer nível abaixar. Uma solução parcial para este problema é o estabelecimento de uma pequena comunicação entre os reservatórios, tornando o balanceamento mais fácil (3). Outra alternativa foi um circuito fechado que incorpora bolsas em vez de reservatórios ${ }^{(10)}$, conferindo uma proteção contra o bombeamento de ar, pois o colapso das bolsas, na ausência de sangue, causa obstrução do circuito. Não obstante, todos estes métodos requerem a inserção de 4 cânulas (em tronco pulmonar, aorta e átrios) e observação cuidadosa do débito em ambas as bombas.

Para evitar esses inconvenientes, decidimos estudar experimentalmente um circuito que emprega uma única bomba centrífuga, seguindo o princípio testado com êxito em um ensaio clínico feito por MORAES et al. ${ }^{(9)}$, em 1978, usando uma bomba de rolete. 
O artifício que permite eliminação da bomba do lado direito consiste em elevar o gradiente transpulmonar, aumentando a pressão no átrio direito e diminuindo-a no átrio esquerdo. A pressão atrial direita é aumentada por expansão de volume de sangue circulante. MORAES et al. (9) preconizam também a posição de Trendelenburg à 30 graus. A pressão atrial esquerda é diminuída coletando-se o sangue das veias pulmonares em um reservatório abaixo do nível do paciente originando um efeito de sifão.

Um cuidado especial deve-se ter na manipulação do átrio esquerdo, como foi previamente documentado (14). Para assegurar drenagem apropriada dessa câmara usamos um tubo de polivinil com uma gaiola (semeIhante a uma válvula de Starr) acoplada à extremidade. Para descomprimir o ventrículo esquerdo usamos um cateter de $2 \mathrm{~mm}$ através do ápice cardíaco. Tentamos evitar esse procedimento com o uso de uma cânula passando através da valva mitral até o ventrículo esquerdo, mas o exame visual após o sacrifício de três animais mostrou dano ao endocárdio por sucção. Uma cânula melhor para descompressão do ventrículo esquerdo precisaria ser aperfeiçoada.

No circuito com dois reservatórios é exigida cautela especial ao se infundir os substitutos sangüíneos do reservatório do lado direito na circulação pulmonar, desde que uma carga de solução de cristalóides pode produzir edema pulmonar ${ }^{(11)}$. O presente circuito permite expansão de volume com substitutos sangüíneos, desde que o volume extracorpóreo entra primeiro na circulação sistêmica, antes de alcançar os pulmões.

A mobilidade do coração encontrada nesta preparação foi semelhante à encontrada em CEC convencional, permitindo um bom acesso a todas as artérias coronárias ${ }^{(9)}$ e a expansão dos pulmões não atrapaIhou a atividade dos cirurgiões ${ }^{(13)}$. Em três animais exe- cutamos com facilidade e bom resultado a anastomose entre a artéria torácica interna e a artéria interventricular anterior, que possui menos de $1 \mathrm{~mm}$ de diâmetro.

Utilizamos a fibrilação ventricular para obter a redução dos movimentos cardíacos a fim de tornar mais simples o experimento, já que a proteção miocárdica não era o objetivo fundamental do estudo. É claro que empregando-se a OA nas operações em humanos será conveniente uma proteção miocárdica mais eficiente que a fibrilação - como a cardioplegia, por exemplo.

Em que pesem as vantagens do procedimento ora proposto, lembremos seja necessário ter em mente que em casos onde a resistência vascular pulmonar é previsivelmente alta, seria mais seguro usar CEC convencional ou um circuito com duas bombas, desde que as pressões transpulmonares providas pelo circuito proposto podem não ser suficientes para assegurar um fluxo de sangue adequado.

\section{CONCLUSÃO}

Este circuito simplificado com bomba única para CEC é capaz de sustentar parâmetros hemodinâmicos e gasométricos adequados enquanto realiza uma OA. Estabelecer e executar a CEC com exclusão apenas do ventrículo esquerdo é rápido e o campo operacional é excelente para intervenções no coração fechado, além da mobilidade do órgão e 0 acesso às artérias coronárias serem semelhantes àquelas proporcionadas pela CEC convencional, com exclusão cardiopulmonar. $O$ procedimento parece fácil, seguro e satisfatório para revascularizações do miocárdio, tornando-se uma alternativa a ser considerada, necessitando investigações clínicas adicionais.

RBCCV 44205-586

Marques E, Cestari I N, Cestari I A, Leirner A A - A simple circuit with only centrifugal pump for extracorporeal circulation with autogenous oxygenation. Rev Bras Cir Cardiovasc 2002; 17(2): 51-56.

ABSTRACT: It was tested in 30 dogs a circuit capable to allow extracorporeal circulation (ECC) with autogenous oxygenation (AO) of the blood employing an only centrifugal pump. With this assembly is unnecessary a pump to the right side: the gradient of transpulmonary pressure was obtained by increasing the pulmonary artery pressure by volemic expansion and decreasing the left atrial pressure by draining this camera by means of a siphon. The heart was electrically fibrillated in the beginning of the bypass and defibrillated in the end. This circuit allowed the maintenance of normal hemodynamic parameters and normal blood gases level during ECC. The operative field and the mobility of the heart were similar to those provided by conventional CEC. We concluded that the use of an only centrifugal pump simplifies ECC with AO, turning it a practical choice for the procedures of myocardial revascularization.

DESCRIPTORS: Extracorporeal circulation, methods. Cardiac surgical procedures, methods. 


\section{REFERÊNCIAS BIBLIOGRÁFICAS}

1. Bjork VD - An effective blood heat exchanger for deep hypothermia in association with extracorporeal circulation but excluding the oxygenator. $J$ Thorac Cardiovasc Surg 1960; 40: 237-52.

2. Blanco G, Oca C, Laguna S, Nunez LE, Schaefer $\mathrm{J}$, Bailey CP - Autogenous lung oxygenation during cardiac bypass. Experimental studies and clinical application in aortic valve surgery. Am J Cardiol 1958; 2: $302-14$.

3. Cass M H \& Ross DN - The evolution of a bypass technique using the lungs as an oxygenator. Guy's Hospital Reports 1959; 108:237-44.

4. Drew CE, Keen G, Benazon DB - Profound hypothermia. Lancet 1959; 1:745-7.

5. Shabetai $\mathrm{R}$ - Cardiac bypass without an artificial lung. Am Heart J 1960; 60: 482-3.

6. Wesolowski AS \& Welch CS - Experimental maintenance of the circulation by mechanical pumps. Surg 1952; 31: 769-93.

7. Berglin E, Sandin O, Winstedt P, William-Olsson $G$ - Extracorporeal circulation without an oxygenator in coronary bypass grafting. J Thorac Cardiovasc Surg 1986; 92: 306-8.

8. Wojnar J, Bochenek A, Wnuk-Wojnar AM, Religa Z, Holowiecki J - Haemocompatibility of extracorporeal circulation technique with autooxygenation: influence of platelet function and homologous blood requirement. Folia Haematol Int Mag Klin Morphol Blutforsch 1990;117: 875-85.

9. Moraes DJ, Abilio FM, Cunha M et al. - Cirurgia de revascularização miocárdica sem oxigenador artificial. J Bras Med 1978; 75-86.

10. Marques E, Oshiro MS, Cestari IA, Hayashida $S A$, Leirner AA - Extracorporeal circulation with autogenous oxygenation: hemodynamic and gasometric parameters. Artif Organs 1995; 19: 470-4.
11. Glenville B - Cardiac surgery using the patient's lungs as the oxygenator. Perfusion 1987; 2:161-6.

12. Bodnar E \& Ross DN - Bilateral cardiac bypass without an oxygenator for coronary artery surgery. Prog Artif Organs 1983:379-82.

13. Read RD, George VP, Cohen M, Lillehei CW Cardiac bypass using autogenous lung for oxygenation. Surgery 1956; 40:840-6.

14. Helmsworth JA, Shabetai R, Cole WR, Neely JC, Albers JE, Gonzales LL - A method of cardiac bypass with autogenous oxygenation. Surgery 1959; 45:129-37.

15. Faymonville ME, Deby-Dupont G, Larbuisson R et al. - Prostaglandin E2, prostacyclin and thromboxane changes during nonpulsatile cardiopulmonary bypass in humans. J Thorac Cardiovasc Surg 1986; 91: 85866.

16. Bolanowsky PJ, Bauer J, Machiedo G, Neville WE - Prostaglandin influence on pulmonary intravascular leukocytic aggregation during cardiopulmonary bypass. J Thorac Cardiovasc Surg 1977; 73:221-4.

17. Kuratani $T$, Matsuda $H$, Sawa $Y$, Kaneko $M$, Nakano S, Kawashima $Y$ - Experimental study in a rabbit model of ischemia-reperfusion lung injury during cardiopulmonary bypass. J Thorac Cardiovasc Surg 1992; 103: 564-8.

18. Chenoweth DE, Cooper SW, Hugli TE, Stewart RW, Blackstone EH, Kirklin JW - Complement activation during cardiopulmonary bypass: evidence for generation of C3a and C5a anaphylatoxins. N Eng $J$ Med 1981; 304: 497-503.

19. Hammerschmidt DE, Stroncek DF, Bowers TK et al. - Complement activation and neutropenia occurring during cardiopulmonary bypass. $J$ Thorac Cardiovasc Surg 1981; 81: 370-7.

20. Westaby S - Mechanisms of membrane damage and surfactant depletion in acute lung injury. Intensive Care Med 1986;12: 2-5. 